\title{
A CORTICAL BASED MODEL OF PERCEPTUAL COMPLETION IN THE ROTO-TRANSLATION SPACE
}

\author{
G. CITTI, A.SARTI
}

Abstract We present a mathematical model of perceptual completion and formation of subjective surfaces, which is at the same time inspired by the architecture of the visual cortex, and is the lifting in the 3-dimensional rototranslation group of the phenomenological variational models based on elastica functional. The initial image is lifted by the simple cells to a surface in the rototraslation group and the completion process is modelled via a diffusion driven motion by curvature. The convergence of the motion to a minimal surface is proved. Results are presented both for modal and amodal completion in classic Kanizsa images.

\section{INTRODUCTION}

1.1. The question of perceptual completion. When we look to the image in Fig. 1, we do not only perceive contours which are characterized by image gradients, but our visual system completes the internal objects, and new contours arise, called "apparent" or "subjective" contours. Gaetano Kanizsa in [33, 34] provided

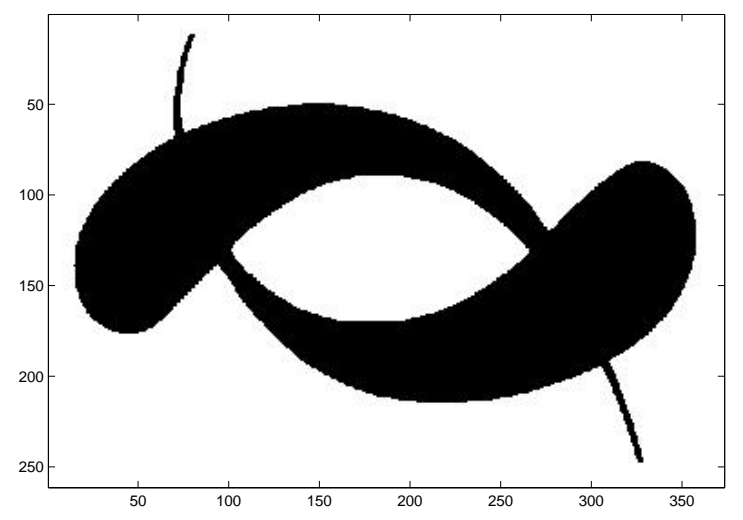

Figure 1. The two fishes of Kanizsa

a taxonomy of perceptual completion phenomena and outlined that they are interesting test to understand how the visual system interpolates existing information ( "modi per andare oltre l'informazione data") and builds the perceived units. He discriminated between "modal completion" and "amodal completion". In the first

Giovanna Citti, Universitá di Bologna, Piazza di Porta S. Donato 5, citti@dm.unibo.it. Alessandro Sarti, Universitá di Bologna, via Risorgimento 2, asarti@deis.unibo.it.

The work was supported by University of Bologna: founds for selected research topics. 
one the interpolated parts of the image are perceived with the full modality of the vision and are phenomenally undistinguishable from real stimuli (this happens for example in the formation of illusory contours and surfaces). In "amodal presence" the configuration is perceived without any sensorial counterpart. Amodal completion is evoked every time one reconstructs the shape of a partially occluded object. Thus it is at the base of the most primitive perceptual configuration that is the segmentation of figure and ground.

Mathematical models of perceptual completion take into account main phenomenological properties as described by psychology of Gestalt. Since subjective boundaries could be linear or curvilinear, their reconstruction is classically performed minimizing the elastica functional

$$
\int_{\gamma}\left(1+k^{2}\right) d s
$$

where the integral is computed on the missed boundary, and $k$ is its curvature (see [44]). The extension of this functional to the level set of the image $I$, has been applied in problems of inpainting (that can be considered a particular case of modal completion) by [45], [1] :

$$
\int_{\Omega}|\nabla I|\left(1+\left|\operatorname{div}\left(\frac{\nabla I}{|\nabla I|}\right)\right|^{2}\right) d x d y
$$

where the integral is extended to the domain of the image. In this way each level line of the image is completed either linearly or curvilinearly as elastica curve. In order to make occluded and occluding objects present at the same time in the image, in [44] (and then in [5], [14]) a third dimension is introduced, and the objects present in the image are represented as a stack of sets, ordered by depth. In [58] the third added dimension is represented by the time, and the algorithm first detects occluding objects, then occluded ones. However these representations fail where a clear depth ordering is not present in the image as in figure 1. In all the past work authors outline the difficulty to treat a functional depending on the curvature, which is a second order operator. Approximation by more tractable functionals has been proposed by [13]. In [6] the associated evolution equation was splitted in two equations, each one of the first order, and depending on two different variables: the image $I$, and the direction of its gradient $\nu=\nabla I /|\nabla I|$.

From the neurophysiological point of view, there is considerable evidence that these kinds of perceptual completion phenomena are accomplished by the first layer of the visual cortex by actively filling in the missing information. The dominant thinking is that there are two cascade mechanism, the first one extracting the existing information ('real' boundaries, image gradients and complex features) by way of feed-forward filtering and the second one completing the missing information with recursive circuitry (even if in the past also feed forward mechanisms for completion have been proposed). The first mechanism is accomplished especially by simple cells in the primary cortex and extracts information about module and orientation of the brightness gradient of the visual stimulus. Not only strong discontinuities corresponding to object boundaries, but also smooth gradients are estimated in this step. The second mechanism propagates extracted information in an orientation specific modality by means of long-range horizontal connections (Field et al. in [26], Kovacs and Julesz, in [38, 39], Kapadia et al, in [35], Gilbert et al., [23]). In this setting the formation of subjective contours is explained as the meeting of 
two neural activation flows shooted by the boundary inducers and closing missing information between the existing boundaries. The specificity of this information propagation is described by the "association fields" (Field, Heyes,Hess, [26]) that indicate boundary collinear directions as privileged diffusion directions to the detriment of orthogonal ones. Petitot and Tondut [54] outlines that the mechanism of boundary completion is not distinct from the mechanism of unification of real contours responsible of transforming fragmental information into continuous curves.

The perspective taken by Petitot and Tondut in [54] is particularly interesting because the perceptual completion problem is considered as a problem of naturalizing phenomenological models on the basis of biological and neurophysiological evidence. They start from the consideration that orientation sensitive simple cells induce a fibration of orientations and that the natural space in which completion is performed is the 3-dimensional image-orientation manifold. Consequently the second order functional (1) is replaced by a first order length minimizing functional in the natural contact structure induced in the 3D space. We further develop this point of view, and build up a model based on lifting of level lines, outlining its feature to segment simultaneously occluding and occluded objects without any depth ordering. Modal and amodal completion can be accomplished in this setting.

The considered image defines a function $I: D \rightarrow \mathbb{R}$, and the points of the domain $D$ are denoted as $(x, y)$. At every point we detect the the normal direction

$$
\nabla I /|\nabla I|=(-\sin (\theta), \cos (\theta))
$$

and lift the domain $D$ to a surface in the 3 -D dimensional space $\Sigma=\{(x, y, \theta(x, y))\}$. On this surface we lift the function $I$ and its gradient:

$$
u(x, y, \theta(x, y))=|\nabla I(x, y)|
$$

defined on $\Sigma$. This first process models the extraction of existing information operated by simple cells in the primary cortex, according to biological models.
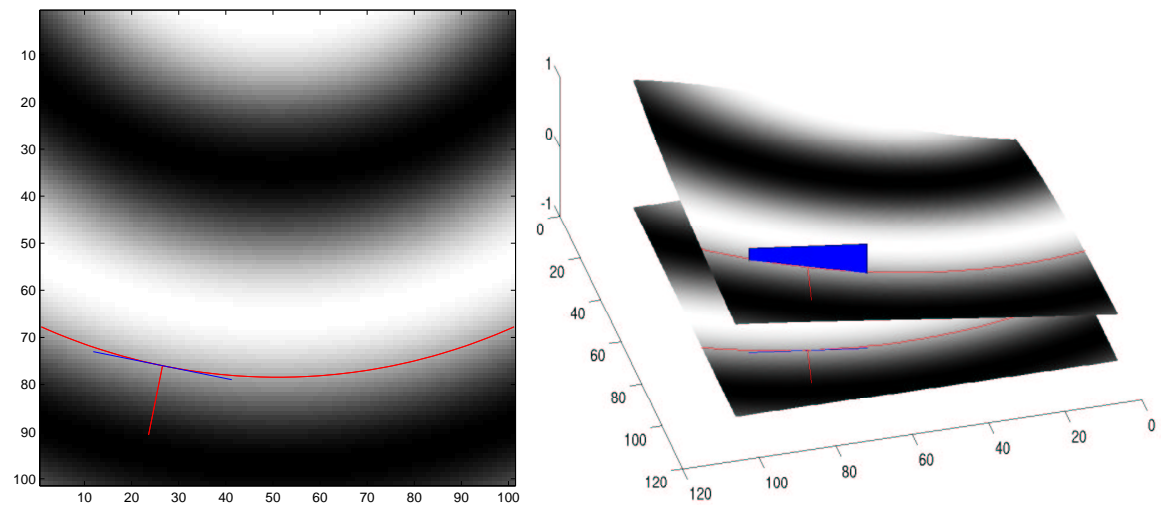

FIGURE 2. The lifting of a whole image and the visualization of a contact plane

Level lines of $u$ lie on the plane orthogonal to the gradient, so that they are tangent at every point to the vector fields

$$
X_{1}=\cos (\theta) \partial_{x}+\sin (\theta) \partial_{y}, \quad X_{2}=\partial_{\theta} .
$$


These are the generators of the Lie algebra of rototranslation, whose natural geometry models the circuitry of the first layers of visual cortex.

The completion of missing boundaries is performed in this setting, via a diffusionconcentration process, which models the diffusion in the direction of association fields, and an orientation selectivity process, as second step in the biological models above recalled. The function $u$ can be identified with the density of a dirac mass concentrated on the initial surface $\Sigma$, so that it will be denoted by

$$
u_{0} \delta_{\Sigma_{0}} .
$$

It is evolved through the so called sub-Laplacian operator, (analogous of the Laplace operator in rototraslation group):

$$
\partial_{t} v=\Delta_{R} v \quad v(0)=u_{0} \delta_{\Sigma_{0}} .
$$

At time $t=1$, with a concentration-selectivity process, we built from $v$ a new surface $\Sigma_{1}$, and a new function $u_{1}$. Iterating this process, we approximate respectively the motion by curvature in the rototralastion group, and the evolution via the Laplace Beltrami operator on the surface. The proof is made extending a technique first introduced in the euclidean setting by [2] and [7].

On the other hand a direct computation shows that the curvature of $I$ can be written in terms of the derivatives of the function $v=X_{1} I$. Consequently also the functional (2), which contains the curvature of $I$, can be expressed a new functional defined in terms of the first derivatives of $v$ :

$$
\int_{\Omega}|\nabla I|\left(1+\left|\operatorname{div}\left(\frac{\nabla I}{|\nabla I|}\right)\right|^{2}\right) d x d y=\int_{\Sigma} \sqrt{\left|X_{1} v\right|^{2}+\left|X_{2} v\right|^{2}} .
$$

This integral is extended only on $\Sigma$, but its formal extension to the whole space is

$$
\int_{\mathbb{R}^{2} \times S^{1}}\left|X_{1} v\right|^{2}+\left|X_{2} v\right|^{2}
$$

whose steepest descent equation is the heat equation in the sublaplacian setting. The fact that the lifted image is defined only on a surface justifies the concentration process. Hence our model can be considered as the natural lifting of model [1] to the three dimensional group.

The model takes into account the main phenomenological characteristics of perceptual completion, including the following:

1) the subjective boundaries are geodesic curves in the metric of the space, which are lifting in the 3-D space of the classic elastica curves, so that they can be linear or curvilinear.

2) the modally completed parts of the image (inpainting) are minimal surfaces in the RT space, and they are foliated in curvilinear isolevels, lifting of the models of Ambrosio, Masnu [1], C. Ballester, and al. [6],

3) occluding and occluded objects are present at the same time in the segmentation, but instead to create a stack of depth ordinate objects as in Mumford, Nitzberg, Shiota [44] and Bellettini March [5], the completion is performed in the natural 3-dimensional cotangent fibration of the image, allowing the segmentation of partially overlapped objects without any depth ordering; this feature is required 
for the segmentation of the classic Kanizsa example of figure 1.

The paper is organized as follows:

- In section 2 we present our cortical based model of perceptual completion

- In section 3 we prove that the model expresses in the new space the variational model of Ambrosio Masnou

- In section 4 we provide the proof of the converge result.

- In section 5 we present a numerical scheme to approximate the model equation and provide results by applying the algorithm to classic gestalt images.

\section{The Model}

2.1. Simple cells and directional derivatives. It is well known that in primates the contour extraction is provided by the area $\mathrm{V} 1$ of the visual cortex, which input arrives from the retina with the intermediation of the Lateral Geniculate Nucleus. The area V1 is the place of the visual cortex in which for the first time one finds cells with elongated receptive fields. These present oriented receptive fields and exhibit even or odd symmetric patterns. Figure 3 shows the odd ones. Simple cells can be considered as units sensitive to brightness gradients, independently if the gradient is originated by a boundary or a smooth region [47]. Simple cells are sensitive to space scale, position and orientation of the contrast gradient and moreover to the polarty of the gradient with respects to the elongation axis of the receptive field. Receptive fields of simple cells are modelled usually as convolution kernels of even and odd filters such as Gabor filters (Jones and Palmer [32], Daugman [12], Marcelja [41]), steerable filters (Perona [52]). Grossberg and Mingolla in [25] have interpreted the functionality of odd receptive fields as gradient indicators and even receptive fields as polarity indicators. A Gabor filter with orientation $\theta$ has the expression

$$
G(x, y, \theta)=\frac{1}{2 \pi s^{2}} \exp \left(-\frac{\left(\tilde{x}^{2}+\tilde{y}^{2}\right)}{s^{2}}+i \tilde{y} / s\right)
$$

where

$$
\tilde{x}=x \cos (\theta)+y \sin (\theta) \quad \text { and } \quad \tilde{y}=-x \sin (\theta)+y \cos (\theta) .
$$

We will consider here the imaginary part of the filter, which is its odd part. Condition (4) describes a rotation of the axis of an angle $\theta$, so that the Gabor filters are obtained from a fixed function, via a rotation.
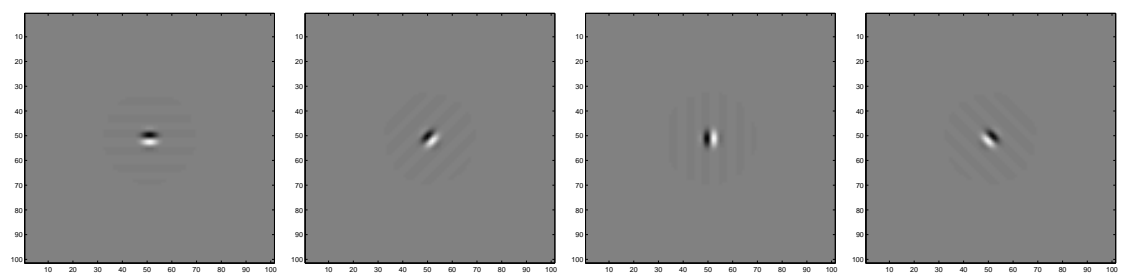

FiguRE 3. Odd part of Gabor filters with different orientations $\theta=0, \theta=\pi / 4, \theta=\pi / 2, \theta=3 / 2 \pi$ 
The odd part of the filter can be locally approximated (up to a multiplicative constant) as

$$
\frac{2 \sin (\tilde{y})}{s^{2}} \exp \left(-\left(\tilde{x}^{2}+\tilde{y}^{2}\right) / s^{2}\right) \simeq \frac{2 \tilde{y}}{s^{2}} \exp \left(-\left(\tilde{x}^{2}+\tilde{y}^{2}\right) / s^{2}\right)=-\partial_{\tilde{y}} \exp \left(-\left(\tilde{x}^{2}+\tilde{y}^{2}\right) / s^{2}\right) .
$$

A derivative in the direction $\tilde{y}$ can be expressed in the original variables $(x, y, \theta)$ as a directional derivative in the direction of the vector $(-\sin (\theta), \cos (\theta))$. We will denote it

$$
X_{3}=-\sin (\theta) \partial_{x}+\cos (\theta) \partial_{y} .
$$

This derivative, applied to a function $I$, expresses the projection of the gradient in the direction $(-\sin (\theta), \cos (\theta))$.

With this notation the filtering generates by convolution with the image I a function

$$
O(x, y, \theta)=-X_{3} \exp \left(-\left(\tilde{x}^{2}+\tilde{y}^{2}\right) / s\right) * I=-X_{3}(\theta) I_{s},
$$

where we have denoted $I_{s}$ the convolution of $I$ with a smoothing kernel:

$$
I_{s}=I * \exp \left(-\left(\tilde{x}^{2}+\tilde{y}^{2}\right) / s\right) .
$$

Note that $O(x, y, \theta)$ depends on the orientation $\theta$. Due to the expression of the Gabor filter, the function $O$ exponentially decays from its maxima. Hence for $\theta$ fixed it selects a neighborhood of the points where the component of the gradient in the direction $(-\sin (\theta), \cos (\theta)$ ), is sufficiently big (see Figure 4).

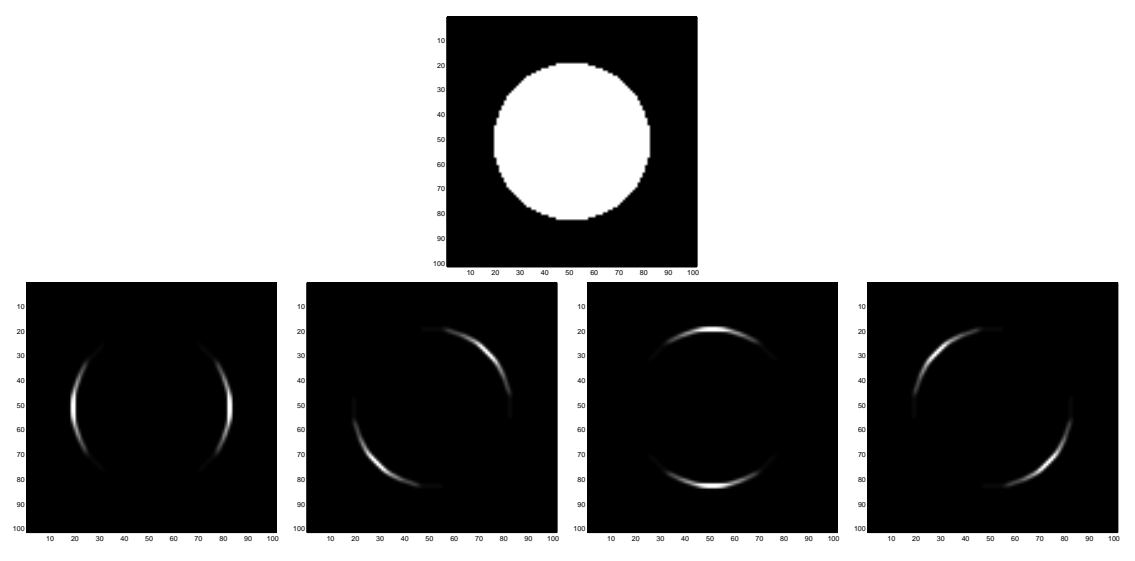

FIgURE 4. The original image showing a white disk (upper) and a sequence of convolutions with different orientations Gabor filters.

Then we can identify the function $O$ with the fiber over the point $(x, y)$ of all the directional derivatives (see Figure 5). This is in agreement with the fundamental idea that the visual cortex assigns a collection of tangent vectors to the points of the image I (Hoffman Ferraro [28]). As outlined in [54] each fiber is physiologically implemented by a hypercolumn of orientations, that is the basic structure of the visual cortex [31]. 

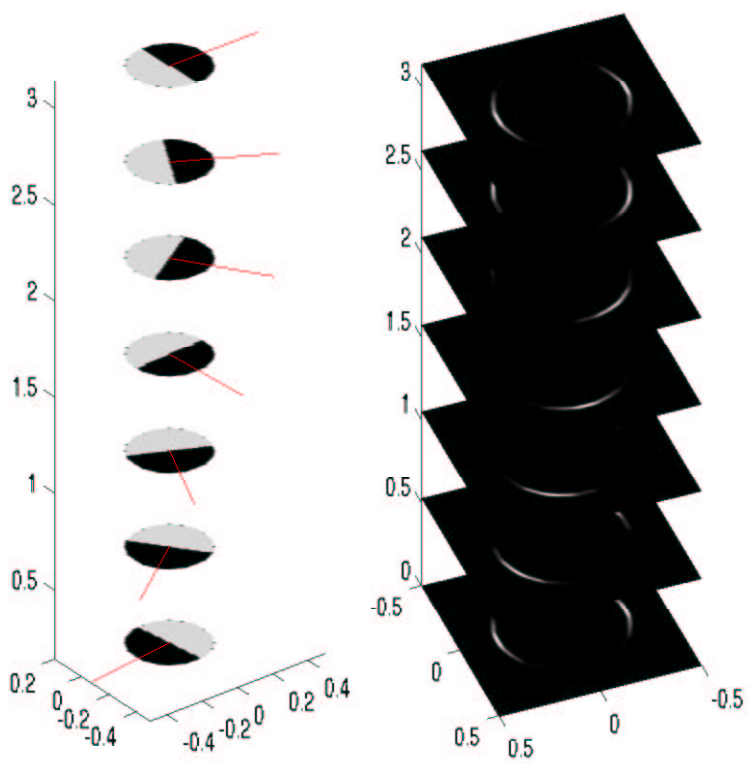

FiguRE 5. Schemata of simple cells arranged in a hypercolumn of orientations (left), and the function $O(x, y, \theta)$, obtained by Eq. (6) as convolution of the image with hypercolumns of orientations (right)

2.2. Orientation selectivity and "non maximal" suppression. The convolution mechansim (6) (that is known by neurophysiologists as orientation bias of the thalamic input) is insufficient to explain the strong orientation tuning exhibited by most simple cells. For these reasons, the classic feedforward mechanism must be integrated with additional mechanisms, in order to provide the sharp tuning experimentally observed. In the past years several models have been presented to explain the emergence of orientation selectivity in the primary visual cortex. These models use different combinations of feedforward (thalamic) and feedback (intracortical) inputs and consider different involvement of excitatory and inhibitory short range connections (Miller, Worgotter, Carandini, Bar, Priebe, Shelley, Nelson $[46,68,10,3,56,60,49])$. Even if the basic mechanism producing strong orientation selectivity is controversial ("push-pull" models $[46,56]$, "emergent" models [49], "recurrent" models [60] only to cite a few), nevertheless it is evident that the intracortical circuitry is able to filter out all the spurious directions and to strictly keep the direction of maximum response of the simple cells. Since $X_{3} I_{\sigma}$ is the projection of the gradient in the direction of the vector $(-\sin (\theta), \cos (\theta))$, the maximum will be achieved at a value $\bar{\theta}$, which is the direction of the gradient.

Then, if we call the point of maximum $\bar{\theta}$, we get

$$
\left|X_{3}(\bar{\theta})\right|=\max _{\theta}\left|X_{3}(\theta)\right| \text {. }
$$

Besides only strict maxima are selected, so that $\left|X_{3}(\bar{\theta}) I_{s}\right|>0$. In this process each point $(x, y)$ in the $2 \mathrm{D}$ domain of the image is lifted to the point $(x, y, \bar{\theta})$. If we denote $T_{(x, y)}\left(\mathbb{R}^{2}\right)$ the tangent space to $\mathbb{R}^{2}$ at the point $(x, y)$, the vector 

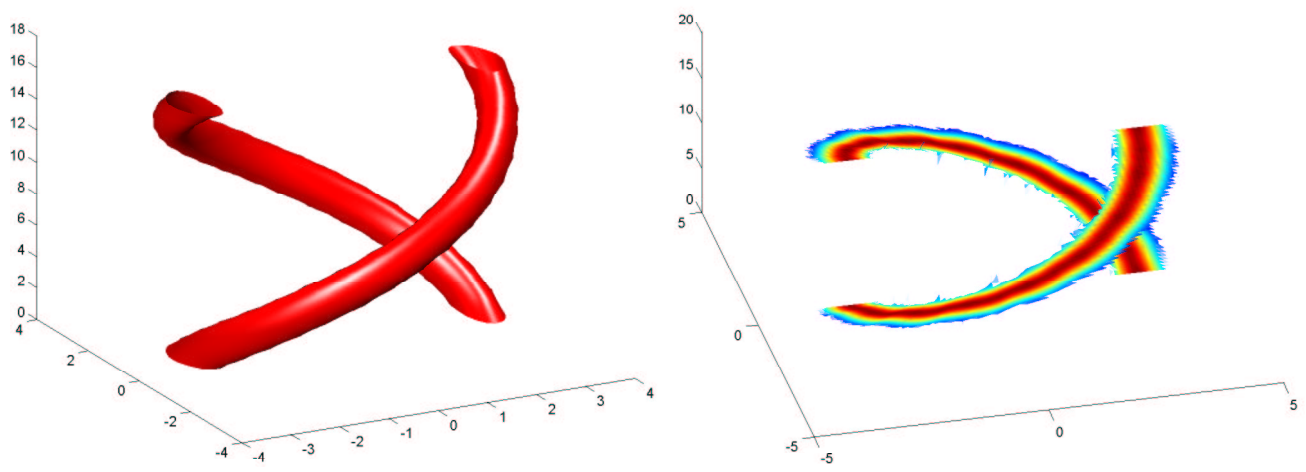

Figure 6. A level set of the function $O(x, y, \theta)$ (left) and the resulting surface after non maximal suppression, called lifted surface (right).

$(-\sin (\bar{\theta}), \cos (\bar{\theta})) \in T_{(x, y)}\left(\mathbb{R}^{2}\right)$ is lifted to the vector field

$$
\vec{X}_{3}(\bar{\theta})=(-\sin (\bar{\theta}), \cos (\bar{\theta}), 0) \in T_{(x, y, \theta)}\left(\mathbb{R}^{2} \times S^{1}\right) .
$$

The whole image domain is lifted to:

$$
\Sigma=\left\{(x, y, \bar{\theta}):\left|X_{3}(\bar{\theta}) I_{s}\right|=\max _{\theta}\left|X_{3}(\theta) I_{s}\right|>0\right\} .
$$

This lifted set corresponds to the maximum of activity of the output of the simple cells, and can be modelled as a dirac mass concentrated on $\Sigma$ itself, with a density $u$, given by the value of the activity:

$$
u(x, y, \theta)=O(x, y, \bar{\theta}) \delta_{\Sigma}
$$

2.3. Non commutative Lie algebra. In the standard euclidean setting, the tangent space to $\mathbb{R}^{2} \times S^{1}$ has dimension 3 at every point. Here we have selected a section $\vec{X}_{3}$ of the tangent space. This defines also a bidimensional subset of the tangent space at every point, orthogonal to $\vec{X}_{3}(\theta)$. This is called horizontal plane, it is generated by

$$
\vec{X}_{1}(\theta)=(\cos (\theta), \sin (\theta), 0), \quad \vec{X}_{2}=(0,0,1),
$$

and it can be represented as

$$
\pi_{x, y, \theta}=\left\{\alpha_{1} \vec{X}_{1}+\alpha_{2} \vec{X}_{2}: \alpha_{1}, \alpha_{2} \in \mathbb{R}\right\} .
$$

This plane, naturally defines a Lie algebra. (see [67]) Indeed to any vector field we can naturally associate the directional derivative in the direction of the vector. We will denote respectively:

$$
X_{1}=\cos (\theta) \partial_{x}+\sin (\theta) \partial_{y}, \quad X_{2}=\partial_{\theta} .
$$

Due to the natural relation between vectors and directional derivatives, the vector $\vec{X}_{i}$ is sometimes identified with the derivative $X_{i}$, but we keep them distinct here for reader convenience. If we choice $\alpha_{1}, \alpha_{2} \in \mathbb{R}$, the linear combination $\alpha_{1} X_{1}+\alpha_{2} X_{2}$ 


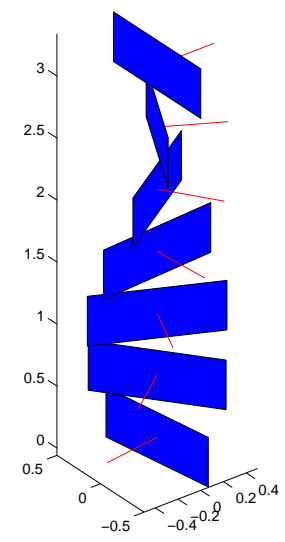

FigURE 7. The contact planes at every point, and the orthogonal vector $\vec{X}_{3}$

defines a new directional derivative. Besides the set of directional derivatives is endowed with an other natural operation, called the bracket (or commuatator). Given two directional derivatives $Y, Z$ their commutator acts on function $u$ as follows:

$$
[Y, Z] u=(Y Z-Z Y) u \text {. }
$$

Note that if $Y$ and $Z$ were partial derivatives, then $[Y, Z]=0$, while this does not happens for directional derivatives. In particular we have

$$
\left[X_{1}, X_{2}\right]=-X_{3} \text {. }
$$

Then we say that $X_{1}$ and $X_{2}$ do not commute. Note, that even thought we have applied two derivatives to the function $u$, the bracket is a first order directional derivative. Then it is possible to define Lie algebra generated by $X_{1}$ and $X_{2}$ as the set of all directional derivatives, which can be represented as linear combination of $X_{1}, X_{2}$ and their commutators of any order. This set is denoted

$$
\mathcal{L}\left(X_{1}, X_{2}\right),
$$

and contains $X_{1}, X_{2}$, and $X_{3}$. Hence, even though the Lie algebra has only 2 generators, it contains the whole 3 dimensional tangent space at every point.

This property is crucial in a connectivity process, which allows to connect different lifted points we refer to [67] for the main properties of the Lie groups.

2.4. Association fields and metric of the rototraslation group. The lifted points of the image would remain isolated without an integrative process allowing to connect local tangent vectors to form integral curves. This process is at the base of both regular contours and illusory contours formation [54].

The most plausible model of connection is based on a mechanism of "local induction". The specificity of this local induction is described by the association field (Field [26]).

The anatomical network of horizontal long-range connections has been proposed as the implementation of association fields, i.e. the magnitude of synaptic interactions depend upon the positions and orientations of the target cells accordingly to the association field. These allow to connect different points of the cortex. 


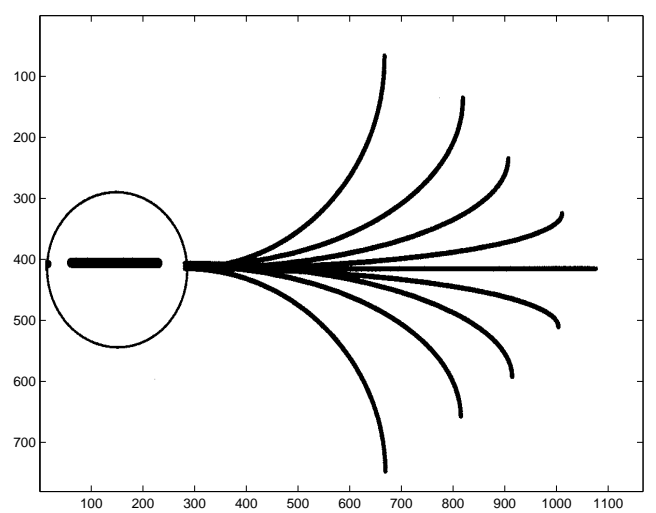

FiguRE 8. Association fields from the experiment of Field,Heyes and Hess
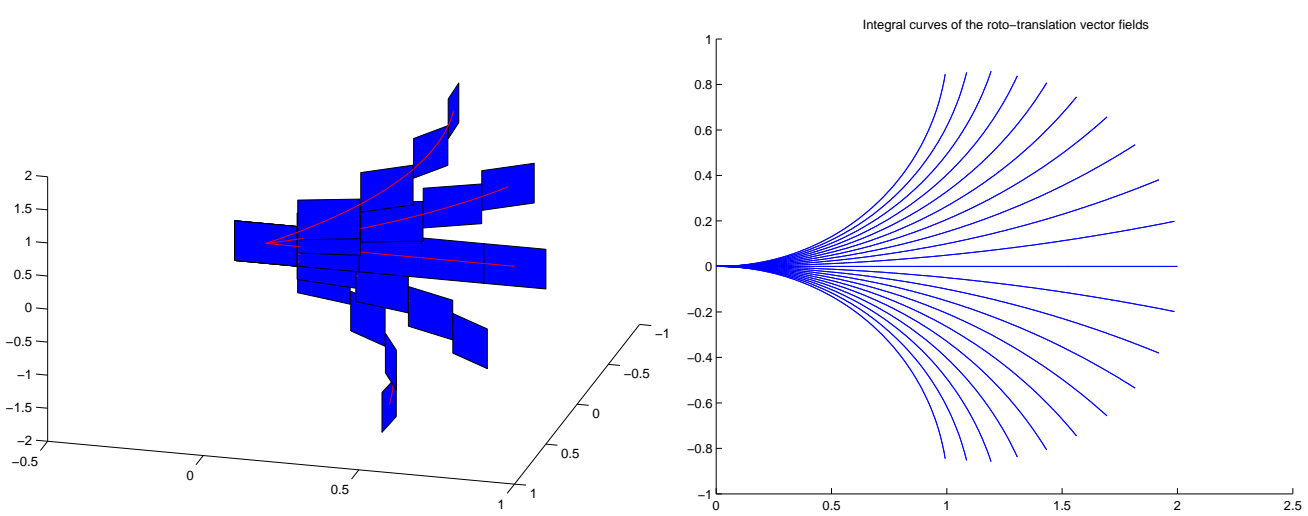

FIGURE 9. Integral curves of the fields by varying the parameter $k$. On the left a $3 \mathrm{D}$ representation with contact planes is shown, in the right its projection onto the image plane is visualized

The local association field is shown in fig. (8) and it can be interpreted as a family of integral curves of the vector fields $X_{1}$ and $X_{2}$, starting at a fixed point $(x, y, \theta)$ :

$$
\gamma^{\prime}(t)=\vec{X}_{1}(\gamma)+k \vec{X}_{2}(\gamma), \quad \gamma(0)=(x, y, \theta),
$$

obtained by varying the parameter $k$ in $\mathbb{R}$ (fig. (9)). For $k$ fixed this curve is tangent to the horizontal plane at every point, it is called horizontal curve and it is denoted

$$
\gamma(t)=\exp \left(t\left(\vec{X}_{1}+k \vec{X}_{2}\right)\right)(x, y, \theta)
$$

Note that the coefficient of $\vec{X}_{1}$ never vanishes in this representation, since the projection on the 2dimensional plane $(x, y)$ of an integral curve on $\vec{X}_{2}$ would be a point. 
Long-range connections can consequently been modelled as admissible curves, but with piecewise constant coefficients $k$. These curves satisfy the so called connectivity property. Indeed the Chow theorem (see [4]) ensures that, since the dimension of the Lie algebra generated by $X_{1}$ and $X_{2}$ is 3 at every point, then for every couple of points $(x, y, \theta),(\bar{x}, \bar{y}, \bar{\theta})$ there exists an horizontal curve $\gamma$ which connects them.

This property, which we deduced from the behavior of the directional derivatives, can be equivalently read on the integral curves. Indeed, starting from a point $(0,0,0)$, and moving along the two curves

$$
\gamma_{1}(t)=\exp \left(t \vec{X}_{2}\right) \exp \left(t \vec{X}_{1}\right)(0,0,0) \quad \gamma_{2}(t)=\exp \left(t \vec{X}_{1}\right) \exp \left(t \vec{X}_{2}\right)(0,0,0)
$$

we reach completely different points $(x, y, \theta),(\bar{x}, \bar{y}, \bar{\theta})$ at the time $t$. Besides the difference between the two points reached in this way is of the form

$$
\exp \left(t^{2} \vec{X}_{3}+o\left(t^{2}\right)\right)(\bar{x}, \bar{y}, \bar{\theta})
$$

so that it is an integral curve of the vector $\vec{X}_{3}$, starting at $(\bar{x}, \bar{y}, \bar{\theta})$.

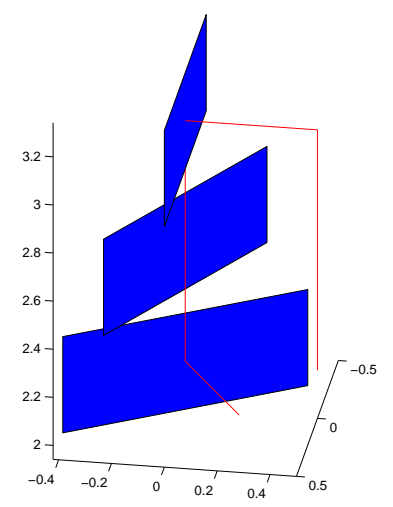

Figure 10. The composition of two integral curves of the rototranslation group is non commutative, depending on the order of application of the operators

Choosing the euclidean metric on the horizontal space, we can set

$$
\left\|\vec{X}_{1}+k \vec{X}_{2}\right\|_{E}=\sqrt{1+k^{2}}
$$

so that we can call length of any curve $\gamma$ expressed as in (10)

$$
\lambda(\gamma)=\int_{0}^{1}\left\|\gamma^{\prime}(t)\right\| d t=\int_{0}^{1} \sqrt{1+\|^{2}}
$$

Consequently it is possible to define

$$
\begin{gathered}
d((x, y, \theta),(\bar{x}, \bar{y}, \bar{\theta}))=\inf \{\lambda(\gamma): \gamma \text { is an horizontal curve } \\
\text { connecting }(x, y, \theta) \text { and }(\bar{x}, \bar{y}, \bar{\theta})\},
\end{gathered}
$$

see [48]. In the Euclidean case the infimum is realized by a geodetic that is a segment. Also here the an horizontal path on which the infimum is achieved is 
called a geodesic, but it can be curvilinear or linear. We will denote ball of center $(\bar{x}, \bar{y}, \bar{\theta})$ and radius $r$ in the metric $d$

$$
B((\bar{x}, \bar{y}, \bar{\theta}), r)=\{(x, y, \theta): d((x, y, \theta),(\bar{x}, \bar{y}, \bar{\theta}))<r\} .
$$

2.5. Local and global invariance properties. Via the exponential mapping the properties of the Lie algebra are carried on the Lie group $\mathbb{R}^{2} \times S^{1}$. Since $X_{1}, X_{2} X_{3}$ are linearly independent at every point, each couple of points can be also connected with integral curves of these 3 vector fields with constant coefficients. In particular each point can be written in the form:

$$
(x, y, \theta)=\exp \left(\alpha_{1} \vec{X}_{1}+\alpha_{2} \vec{X}_{2}+\alpha_{3} \vec{X}_{3}\right)(0,0,0),
$$

where $\left(\alpha_{1}, \alpha_{2}, \alpha_{3}\right)$ are constant. Up to a first order approximation $\alpha_{i}$ are the projection in the directin of the vector fields $\vec{X}_{i}$ of the point $(x, y, \theta)$. This means that

$$
\alpha_{1} \simeq x \cos (\theta)+y \sin (\theta) \quad \alpha_{1} \simeq \theta, \quad \alpha_{3} \simeq-x \sin (\theta)+y \cos (\theta) .
$$

It has been proved in [48] that the distance $d$, defined in (11) in terms of two vector fields, is locally equivalent to

(12) $d((x, y, \theta),(0,0,0)) \simeq\left((x \cos (\theta)+y \sin (\theta))^{2}+\theta^{2}\right)^{1 / 2}+|x \sin (\theta)-y \cos (\theta)|^{1 / 2}$.

This means that $d$ is linear in the directions $\vec{X}_{1}$ and $\vec{X}_{2}$, which define the contact plane, and behave as a square root in the direction $\vec{X}_{3}$ of the commutator. In other words for $r$ small the balls are elongated in the directions $\vec{X}_{1}$ and $\vec{X}_{2}$, and thinner in the direction $\vec{X}_{3}$.

The natural dilation defined locally around $(0,0,0)$, is then

$$
\delta_{\lambda}\left(\alpha_{1}, \alpha_{2}, \alpha_{3}\right)=\left(\lambda \alpha_{1}, \lambda \alpha_{2}, \lambda^{2} \alpha_{3}\right)
$$

In particular this dilation sends the ball $B((0,0,0), 1)$ in the ball with the same center and radius $\lambda$. Analogously it is possible to locally define dilations around any fixed point $(x, y, \theta)$.

The exponential mapping also define a composition law, which will play the same role of the sum in the euclidean setting:

$$
\left(x_{1}, y_{1}, \theta_{1}\right)+_{R}\left(x_{0}, y_{0}, \theta_{0}\right)=\left(x_{2}, y_{2}, \theta_{2}\right)
$$

where

$$
\left(x_{2}, y_{2}, \theta_{2}\right)=\left(\cos \left(\theta_{1}\right) x_{0}-\sin \left(\theta_{1}\right) y_{0}+x_{1}, \sin \left(\theta_{1}\right) x_{0}+\cos \left(\theta_{1}\right) y_{0}+y_{1}, \theta_{0}+\theta_{1}\right) .
$$

Since the dilation does not commute with the group law, the dilation is not a global property, and the group is non homogeneous.

The distance and the differentiation on the contrary commute with left translations, indeed

$$
\left.d((\bar{x}, \bar{y}, \bar{\theta}),(x, y, \theta))=d\left(0,-{ }_{R}(\bar{x}, \bar{y}, \bar{\theta})+{ }_{R}(x, y, \theta)\right)\right)
$$

Besides, if we fix $(\bar{x}, \bar{y}, \bar{\theta})$ and call

$$
u_{(\bar{x}, \bar{y}, \bar{\theta})}(x, y, \theta)=u\left((\bar{x}, \bar{y}, \bar{\theta})+_{R}(x, y, \theta)\right)
$$

then

$$
X_{i} u_{(\bar{x}, \bar{y}, \bar{\theta})}=\left(X_{i} u\right)\left((\bar{x}, \bar{y}, \bar{\theta})+_{R}(x, y, \theta)\right) .
$$


2.6. Riemannian approximation of the metric. Note that this metric is not induced by a riemannian metric. Indeed we have defined the euclidean metric only on the horizontal plane. We can extend it on all the tangent space defining norm of a vector as the euclidean norm of its projection on the horizontal tangent space. Precisely for a vector $\vec{v} \in T_{x, y, \theta}\left(\mathbb{R}^{2} \times S^{1}\right)$ represented in the standard basis $\partial_{x}, \partial_{y}, \partial_{\theta}$ we define

$$
\begin{gathered}
|v|_{g}^{2}=\left\|\left(\begin{array}{ccc}
\cos (\theta) & \sin (\theta) & 0 \\
0 & 0 & 1
\end{array}\right)\left(\begin{array}{l}
v_{1} \\
v_{2} \\
v_{3}
\end{array}\right)\right\|_{E}^{2}=\left\|\left(v_{1} \cos (\theta)+v_{2} \sin (\theta), v_{3}\right)\right\|_{E}^{2}= \\
=\left(v_{1} \cos (\theta)+v_{2} \sin (\theta)\right)^{2}+v_{3}^{2}= \\
v_{1}^{2} \cos ^{2}(\theta)+v_{2}^{2} \sin ^{2}(\theta)+v_{1} v_{2} \cos (\theta) \sin (\theta)+v_{3}^{2}
\end{gathered}
$$

Hence we formally obtain an expression for the inverse of the metric:

$$
g^{i j}=\left(\begin{array}{ccc}
\cos ^{2}(\theta) & \sin (\theta) \cos (\theta) & 0 \\
\sin (\theta) \cos (\theta) & \sin ^{2}(\theta) & 0 \\
0 & 0 & 1
\end{array}\right)
$$

Since this matrix is not invertible, be can not induce a riemannian metric on the space. However, we can consider a riemannian approximation of the metric, and add a viscosity term in the direction $X_{3}$. The approximated riemannian norm of the tangent vector $\vec{v}$ will be

$$
|v|_{g}^{2}=\left(\cos (\theta) v_{1}+\sin (\theta) v_{2}\right)^{2}+v_{3}^{2}+\epsilon^{2}\left(-\sin (\theta) v_{1}+\cos (\theta) v_{2}\right)^{2} .
$$

The associated matrix is

$$
g^{i j}{ }_{\epsilon}=\left(\begin{array}{ccc}
\cos ^{2}(\theta)+\epsilon^{2} \sin (\theta) & \left(1-\epsilon^{2}\right) \sin (\theta) \cos (\theta) & 0 \\
\left(1-\epsilon^{2}\right) \sin (\theta) \cos (\theta) & \sin ^{2}(\theta)+\epsilon^{2} \cos ^{2}(\theta) & 0 \\
0 & 0 & 1
\end{array}\right)
$$

This matrix is invertible, and the inverse matrix $g_{i j_{\epsilon}}$ defines a norm on cotangent space at every point, as follows. If $w=\left(w_{1}, w_{2}, w_{3}\right) \in T_{x, y, \theta}^{*}\left(\mathbb{R}^{2} \times S^{1}\right)$

$$
\left|\left(w_{1}, w_{2}, w_{3}\right)\right|_{g, \epsilon}^{2}=\left(\cos (\theta) w_{1}+\sin (\theta) w_{3}\right)^{2}+\theta^{2}+\frac{1}{\epsilon^{2}}(\sin (\theta) x-\cos (\theta) y)^{2} .
$$

The geodesic distance $d_{\epsilon}$ associated to $g_{i j_{\epsilon}}$ tends as $\epsilon$ goes to 0 to the subriemannian one, defined in (11) (see [?]).

2.7. Functions and surfaces regular with respect to the metric induced by the association fields. The metric introduced by the association fields allows to recognize that the lifted sets are regular and surfaces in the Rototraslation space.

In particular in a Lie algebra it is possible to introduce natural derivatives, called Lie derivatives, which are performed along the association fields:

Definition 2.1. If $\gamma_{i}(s)=\exp \left(s X_{i}\right)\left(\xi_{0}\right)$ we define the Lie derivative of a function $u$ in $\xi_{0}$ as

$$
X_{i} u\left(\xi_{0}\right)=\frac{d}{d s}(u \circ \gamma)_{\mid s=0},
$$

when the right hand exists and is finite. 
This derivative concises with the usual one, when $u$ is smooth. Besides it is the derivative implemented by the association fields.

The set of functions $u$ such that $X_{1} u$ and $X_{2} u$ exist and are continuous will be called $C_{R}^{1}$. In this case the gradient of $u$ is the horizontal section

$$
\nabla_{R} u=\left(X_{1} u, X_{2} u\right) .
$$

We explicitly note that we do not require that $u$ is differentiable with respect to $X_{3}$, hence in general $C^{1}$ and $C_{R}^{1}$ are not the same set. On the contrary the function $u$ is only Holder continuous of order $1 / 2$ in the direction $X_{3}$. Analogously $u \in C_{R}^{2}$ if $\nabla u \in C_{R}^{1}$. In particular, if $u \in C^{2}$, by relation (9), is differentiable with respect to $X_{3}$. In other words the derivative $X_{3}$ has to be considered a second order derivative with respect to the structure of the space. We can now define a regular surface:

Definition 2.2 (regular surface). A subset $\Sigma \subset \mathbb{R}^{2} \times S^{1}$ is called a $R$-regular surface if it can be locally described as the 0-level set of a function $u$ of class $C_{R}^{1}$, with non vanishing gradient. Precisely there exists a neighborhood $U$ of every point such that

$$
\Sigma \cap U=\left\{(x, y, \theta) \in U: u(x, y, \theta)=0, \nabla_{R} u(x, y, \theta) \neq 0\right\} .
$$

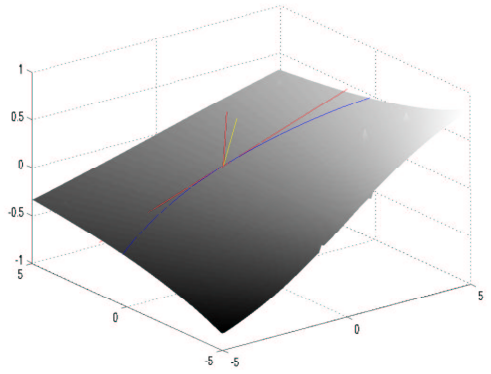

FIGURE 11. normal and tangent vectors to the lifted surface

In particular the image domain, lifted to the set $\Sigma$ as in formula (8), define a regular surface in the rototraslation group. Indeed, we can represent $\Sigma$ as

$$
\Sigma=\left\{(x, y, \theta):\left|X_{3}(\theta) I_{s}\right|>0, X_{2}\left(X_{3}(\theta) I_{s}\right)=0, X_{2}^{2}\left(X_{3}(\theta) I_{s}\right) \neq 0\right\} .
$$

If we call $v=X_{2}\left|X_{3}(\bar{\theta}) I_{s}\right|$, the set $\Sigma$ becomes

$$
\Sigma=\left\{(x, y, \theta): v=0, \quad X_{2} v \neq 0\right\} .
$$

If $\Sigma$ is a regular surface in $\mathbb{R}^{2} \times S^{1}$, we call horizontal normal to $\Sigma$, and denote $\nu_{R}$ the projection on the horizontal plane of the euclidean normal:

$$
\nu_{R}=\frac{\left(X_{1} u, X_{2} u\right)}{\sqrt{\left(X_{1} u\right)^{2}+\left(X_{2} u\right)^{2}}} .
$$

A $R$-horizontal tangent vector is an horizontal vector, tangent to $\Sigma$. Since the normal belongs to the horizonal plane, then there exists exactly a line of tangent vectors, and the expression of the unitary tangent vector will be:

$$
T_{R}=\frac{\left(X_{2} u,-X_{1} u\right)}{\sqrt{\left(X_{1} u\right)^{2}+\left(X_{2} u\right)^{2}}} .
$$


Finally the curvature is defined, in analogy with the euclidean one, as the $R$-divergence of the $R$-normal vector:

$$
H_{R}(\Sigma)=\operatorname{div}_{R}\left(\nu_{R}\right)
$$

where the $R$-divergence of a vector $\left(\nu_{1}, \nu_{2}\right)$ is defined as

$$
\operatorname{div}_{R} \nu=X_{1} \nu_{1}+X_{2} \nu_{2} \text {. }
$$

2.8. Activity propagation in the sub-riemannian space. Up to now we have built up a geometric space inspired by the functional geometry of the primary cortex. In the cortex neural activity develops and propagates itself in this-subriemannian space. For seek of semplicity, in this study we consider an extremely simple model of activity propagation, i.e. a simple linear diffusion in the geometric structure. It exactly means to diffuse with respect to the sublaplacian operator

$$
\partial_{t} u=\Delta_{R} u \text {. }
$$

The sublaplacian operator in this setting is defined, in analogy with the classical laplacian

$$
\Delta_{R} u=\operatorname{div}_{R}\left(\nabla_{R} u\right) .
$$

Analogously if the function $u$ is of class $C^{2}$, the matrix of all the $R$-second derivatives is called Hessian matrix

$$
\text { Hess }_{R} u=\left(\begin{array}{cc}
X_{1}^{2} u & \frac{1}{2}\left(X_{1} X_{2}+X_{2} X_{1}\right) u \\
\frac{1}{2}\left(X_{1} X_{2}+X_{2} X_{1}\right) u & X_{2}^{2} u
\end{array}\right) .
$$

Note that, since $X_{1}$ and $X_{2}$ do not commute, in order to make the matrix symmetric, the coefficients $\left(\text { Hess }_{R}\right)_{12}$, and $\left(\text { Hess }_{R}\right)_{21}$ contains the mean of the mixed derivatives. Also note that the laplacian is the trace of the Hessian.

The laplacian operator is represented as a sum of squares of 2 vector fields in $\mathbb{R}^{2} \times S^{1}$. Hence it is strongly degenerate, since the associated matrix has 0 determinant at every point. However it has been deeply studied after the result of Hörmander in [29]:

Theorem 2.1. Since the Lie algebra generated by $X_{1}$ and $X_{2}$ is of maximum rank at every point, then the sublaplacian operator is hypoelliptic. This simply means that for every initial datum, the solution of the evolution equation is of class $C^{\infty}$

Most of the classical analysis has been carried out in the spaces: existence and local estimates of the fundamental solution has been proved by [57, 48], gaussian estimates by Varopoulos, Saloff-Coste and Coulhon in [66], via semigroup theory, by Kusuoka and Stroock [36, 37] via Gevrey methods. We also refer to [8] for a proof in the homogeneous situation. As a consequence the classical Schauder and Sobolev regularity results have been proved by [19, 20, 21], representation formulas have established, embedding theorems and compactness results. In particular the solution of (16) can be explicitly represented in terms of a fundamental solution:

$$
u((x, y, \theta), t)=\int \Gamma(\zeta, t) u_{0}\left(\zeta_{-}(x, y, \theta)\right) d \zeta
$$

where $u_{0}$ is the initial value, at time $t=0$. Here the convolution is performed using the group law of the space, and the variable $\zeta$ stands for $\left(x^{\prime}, y^{\prime}, \theta^{\prime}\right)$. The expression of $\Gamma$ is not known explicitly, but it can estimated from above and from below as

$$
|\Gamma((x, y, \theta), t)| \leq \frac{C}{t^{Q / 2}} \exp \left(-\frac{d^{2}(x, y, \theta)}{t}\right),
$$


where $Q=4$. In particular the level lines of $\Gamma$ are the balls of the metric, and the diffusion is made along these sets.

\subsection{Completion model and minimal surfaces in the rototranslation space.} The joint work of subriemannian diffusion (15) and non maximal suppression (8) allows to propagate existing information and then to complete boundaries and surfaces. Starting from the lifting

$$
\begin{gathered}
t=0\left\{\begin{array}{l}
u_{0}=O \\
\Sigma_{0}=\left\{(x, y, \theta): \partial_{\theta} u_{0}=0, \partial_{\theta \theta} u_{0}<C\right\}
\end{array}\right. \\
u(\cdot, 0)=u_{0} \delta_{\Sigma_{0}}
\end{gathered}
$$

the two mechanisms are simultaneously applied until the completion is reached. To take into account the simultaneous work of diffusion and non maximal suppression we consider iteratively diffusion in a finite time interval followed by non maximal suppression, and we compute the limit when the time interval tends to 0 .

Indeed, given a function $u_{n}$, whose maxima in a given direction are attained on a surface $\Sigma_{n}$, we diffuse in an interval $[0, h]$ of time the function $u_{n} \delta_{n}$, where $\delta_{n}$ is the $\delta$ function concentrated on the surface $\Sigma_{n}$ :

$$
u_{t}=\Delta w, \quad u=u_{n} \delta_{n} \text { per } t=0 .
$$

Then we define a new surface $\Sigma_{n+1}$ as the 0 level set of the derivative of $u$ in the direction normal to $\Sigma_{n}$, and consider the new function $u_{n+1}=u \delta_{\Sigma_{n+1}}$.

$$
\begin{gathered}
t \in[n h,(n+1) h]\left\{\begin{array}{l}
\partial_{t} u=\Delta_{R} u \text { in }\left(\mathbb{R}^{2} \times S^{1}\right) \backslash \Sigma_{0} \\
u(\cdot, n h)=u_{n}(\cdot, n h) \delta_{\Sigma_{n}}
\end{array}\right. \\
t=(n+1) h\left\{\begin{array}{l}
u_{n+1}(\cdot,(n+1) h)=u(\cdot,(n+1) h) \\
\Sigma_{n+1}((n+1) h)=\left\{\partial_{\nu_{\Sigma_{n}}} u_{n+1}=0, \partial_{\nu_{\Sigma_{n}}}^{2} u_{n+1}<0\right\}
\end{array}\right.
\end{gathered}
$$

If we fix a time $T$, we can choose intervals of length $h=T /(n+1)$, and we get the two sequences: $u_{n+1}(\cdot, T), \Sigma_{n+1}(T)$. In section 5 we will a sketch of the proof of the convergence of the two sequences $\Sigma_{n}(T)$ and $u_{n}(T)$ respectively to mean curvature flow $\Sigma(T)$ of the surface $\Sigma_{0}$ and the beltrami flow on $\Sigma$. For $T \rightarrow+\infty$ the function $\Sigma(T)$ converges to a minimal surface in the rototraslation space, in the sense that it satisfies:

$$
\begin{gathered}
\Delta_{R} u-\left\langle\operatorname{Hess}_{R} u \nu_{\Sigma}, \nu_{\Sigma}\right\rangle=0 \\
\operatorname{div}_{R}\left(\nu_{\Sigma}\right)=0
\end{gathered}
$$

The existence of minimal surfaces in a Carnot group on $\mathbb{R}^{3}$ has been recently proved by [9] and [51]. A definition of motion by curvature in a viscosity sense which extends to the rototraslation group the well known definition of Evans and Spruck has been given by Manfredi in [43]. 


\section{Relation with Phenomenological models}

3.1. Lifted curves and elastica. Petitot and Tondut have already noted in [54] that for a lifted curve $\gamma$ expressed in the form:

$$
\gamma^{\prime}=X_{1}+k X_{2}
$$

the coefficient $k$ is is the curvature of the projection of $\gamma$ on the $(x, y)$ plane.

Indeed if we denote $\gamma(t)=(x(t), y(t), \theta(t))$, by definition of integral curve we have

$$
x^{\prime}=\cos (\theta), \quad y^{\prime}=\sin (\theta), \quad \theta^{\prime}=k
$$

¿From the first two relations it follows that

$$
\theta=\arctan \left(\frac{y^{\prime}}{x^{\prime}}\right)
$$

Differentiating this expression we get

$$
\theta^{\prime}=\sqrt{x^{\prime}+y^{\prime}} \frac{y^{\prime \prime} x^{\prime}-x^{\prime \prime} y^{\prime}}{\left(\left(x^{\prime}\right)^{2}-\left(y^{\prime}\right)^{2}\right)^{3 / 2}} .
$$

In particular the length of $\gamma$ is the elastica functional, suitably modified:

$$
\int \sqrt{\dot{x}^{2}+\dot{y}^{2}+\dot{\theta}^{2}}=\int \sqrt{\dot{x}^{2}+\dot{y}^{2}} \sqrt{1+k^{2}} .
$$

For horizontal curves there exists a notion formally equal to the definition of curvature, but computed with respect to the coordinates in the direction $X_{1}$. Indeed we represent a curve $\gamma$ in the form

$$
\left.\gamma^{\prime}=\gamma_{1}^{\prime} X_{1}+\gamma_{2}^{\prime} X_{2}\right),
$$

the contact curvature is defined as

$$
k_{c}=\frac{\gamma_{2}^{\prime \prime} \gamma_{1}^{\prime}-\gamma_{2}^{\prime} \gamma_{1}^{\prime \prime}}{\left(\left(\gamma_{1}^{\prime}\right)^{2}-\left(\gamma_{2}^{\prime}\right)^{2}\right)^{3 / 2}}
$$

3.2. Lifted surfaces and curves. We have recalled that, if $\Sigma$ is a regular surface, at any point is defined an unique horizontal tangent vector $T_{R}$. Is then possible to consider a curve $\gamma$ on $\Sigma$, and tangent to $T_{R}$, at every point. This curve is obviously horizontal, and can be represented as

$$
\gamma^{\prime}(t)=X_{1}-\frac{X_{1} u}{X_{2} u} X_{2} .
$$

From this relation and (18) it immediately follows that the curvature of the projection of $\gamma$ on the $2 \mathrm{D}$ plane $x, y$ is

$$
k=\frac{-X_{1} u}{X_{2} u} .
$$

A direct verification ensures that

Remark 3.1. The curvature of the surface $\Sigma$ coincides with the contact curvature of the horizontal curve $\gamma$ lying on $\Sigma$.

A lifted surface has been represented in (14) as

$$
\Sigma=\left\{(x, y, \theta): v=0, X_{2} v \neq 0\right\},
$$


hence the Dini theorem ensures that $\Sigma$ locally is the graph of a suitable function $\theta$ defined in an open subset $U$ of $\mathbb{R}^{2}$ :

$$
\Sigma=\{(x, y, \theta(x, y)):(x, y) \in U\},
$$

Then also the measure of the lifted surface is represented in terms of curvature of bidimensional curves:

Proposition 3.1. Let $\Sigma$ a regular admissible surface parametrized by $\theta=\theta(x, y)$. For every point $(x, y, \theta)$ we denote $k$ the euclidean curvature of the projection of the curve $\gamma$, defined on $\Sigma$ in (19). Then the measure of $\Sigma$ is the following one:

$$
\operatorname{mis}(\Sigma)=\int \sqrt{1+k^{2}(\theta(x, y))} d x d y .
$$

3.3. Relation of our model with Morel-Masnou one. Here we show the relation with the level line model of Morel-Masnou, Ambrosio-Masnou who proposed to minimize the functional (2)

A lifted surface has been represented in (14) as

$$
\Sigma=\left\{(x, t, \theta): v=0 \quad X_{2} v \neq 0\right\},
$$

where $v=X_{2} X_{3} I_{\sigma}=X_{1} I_{\sigma}$. On this surface we evolve the function

$$
v=X_{1} I_{s}+\left|X_{3} I_{s}\right| .
$$

Note that this means that we do not consider the intensity of $I_{s}$, but only its level sets. However this function has exactly the same level sets as the function

$$
\tilde{v}=X_{1} I_{s}+I_{s},
$$

which coincides with $I_{s}$ on the surface. A simple differentiation show that (for both $v$ and $\tilde{v})$

$$
\left|X_{1} v\right|=|\nabla I|\left|\operatorname{curv}\left(I_{s}\right)\right| \quad\left|X_{2} v\right|=\left|\nabla I_{s}\right|,
$$

so that substituting in 2 we get

$$
\begin{aligned}
\int\left|\nabla I_{s}\right|\left(1+\left|\operatorname{curv}\left(I_{s}\right)\right|^{2}\right) & =\int \sqrt{\left|\nabla I_{s}\right|\left(1+\left|\operatorname{curv}\left(I_{s}\right)\right|^{2}\right)} \sqrt{\left(1+k^{2}\right)(\theta(x, y))}= \\
& =\int_{v=0} \sqrt{\left(\left|X_{1} v\right|^{2}+\left|X_{2} v\right|^{2}\right)},
\end{aligned}
$$

where $\theta$ is the parametrisation previously introduced. Applying the coarea formula we can extend the integral on the whole space:

$$
\int_{\mathbb{R}^{2} \times S^{1}}\left|X_{1} v\right|^{2}+\left|X_{2} v\right|^{2} \quad \text { if } X_{1} I_{s}=0
$$

This functional is simply the gradient squared, and its associated steepest descent equation is the heat equation. Hence this describes a diffusion. However, since we only want to consider the values on the surface, we also need to concentrate again on the surface. In this sense our model ca be considered a lifting of Masnou Model in $\mathbb{R}^{2} \times S^{1}$. Note that it drastically reduces the complexity of the minimisation problem. 


\section{NUMERICAL SCHEME AND COMPUTATIONAL RESULTS}

In this section, we show how to approximate the model equations with finite differences. Let us consider a rectangular uniform grid in space-time $(t, x, y, \theta)$; then the grid consists of the points $\left(t_{n}, x_{l}, y_{m}, \theta_{q}\right)=(n \Delta t, l \Delta x, m \Delta y, q \Delta \theta)$. The relation between the increments will be deduced from the structure of the local dilations we have defined. in (13). Since the grid is rectangular, while the natural increments are linear in direction $\vec{X}_{1}$ and $\vec{X}_{2}$, and quadratic in direction $\vec{X}_{3}$, we are forced to choice

$$
\Delta x=\Delta y, \quad \Delta \theta=\Delta x^{2} \quad \Delta t=\Delta x^{2} .
$$

Following standard notation, we denote by $u_{l m q}^{n}$ the value of the function $u$ at the grid point $\left(t_{n}, x_{l}, y_{m}, \theta_{q}\right)$. We approximate time derivative with a first order forward difference and space derivative with second order centered scheme. First and second derivatives in the direction of the subriemannian fields are approximated with

$$
\begin{gathered}
D_{1} u_{l m q}^{n}=\cos \left(\theta_{q}\right) D_{x} u_{l m q}^{n}+\sin \left(\theta_{q}\right) D_{y} u_{l m q}^{n} \\
D_{2} u_{l m q}^{n}=D_{\theta} u_{l m q}^{n} \\
D_{11} u_{l m q}^{n}=\cos \left(\theta_{q}\right)^{2} D_{x x} u_{l m q}^{n}+2 \cos \left(\theta_{q}\right) \sin \left(\theta_{q}\right) D_{x y} u_{l m q}^{n}+\sin \left(\theta_{q}\right)^{2} D_{y y} u_{l m q}^{n} \\
D_{22} u_{l m q}^{n}=D_{\theta \theta} u_{l m q}^{n}
\end{gathered}
$$

where $D$ is the usual finite difference operator on the discretized function and the subscripts $x, y, \theta$ indicate the direction of differentiation.

$$
\begin{gathered}
u_{l m q}^{n+1}=u_{l m q}^{n}+\Delta_{t}\left(D_{11} u_{l m q}^{n}+D_{22} u_{l m q}^{n}\right) \\
0 \leq n \leq N_{1} \\
u_{l m q}^{n+1}=u_{l m q}^{n}+\Delta_{t} *\left(\frac{\left(D_{2} v_{l m q}^{n}\right)^{2} D_{11} u_{l m q}^{n}+\left(D_{1} v_{l m q}^{n}\right)^{2} D_{22} u_{l m q}^{n}}{D_{11} v_{l m q}^{n}+D_{22} v_{l m q}^{n}}-\right. \\
\left.-\frac{\left(D_{12} u_{l m q}^{n}+D_{21} u_{l m q}^{n}\right) D_{1} v_{l m q}^{n} D_{2} v_{l m q}^{n}}{D_{11} v_{l m q}^{n}+D_{22} v_{l m q}^{n}}\right) \\
v_{l m q}^{n+1}=D_{2} u_{l m q}^{n} \\
N_{1} \leq n \leq N_{2}
\end{gathered}
$$

We impose Neumann boundary conditions on $x$ and $y$ and periodic boundary conditions on the third direction $\theta$. The time step $\Delta t$ is upper bounded by the CFL (Courant-Friedrich-Levy) condition that ensures the stability of the evolution [40].

In the first numerical experiment we consider the completion of a figure that has been only partially lifted in the roto-translation space. This example mimics the missing information due to the presence of the macula cieca (blind spot) that is modally completed by the human visual system, as outlined in [33]. The original image (see Figure 12), top left) is lifted in the rotranslation space with missing 

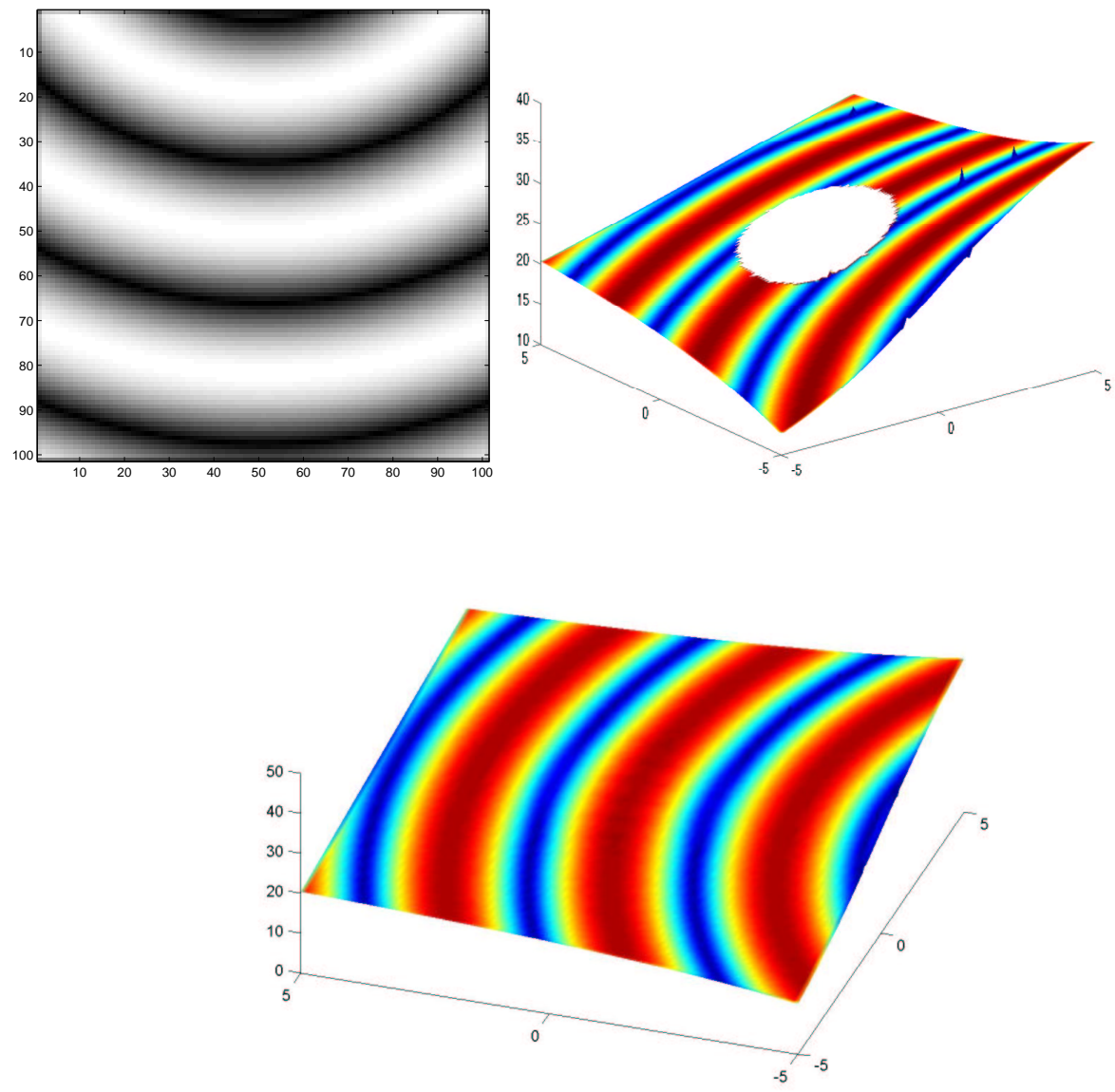

FiguRE 12. The original image (top left) is lifted in the rototranslation space with missing information in the center, like in the phenomenon of macula cieca (top right). The surface is completed by the algorithm (bottom).

information in the center (top right). The lifted surface is completed by iteratively applying eqs until a steady state is achieved. As proved in Theorem 5.1, the final surface is minimal with respects to the subriemannian metric.

In Figure 13 an occlusion problem is considered. The initial image (top left) shows an underlying object partially occluded by a vertical stripe. The human visual system contemporary segments the occluding object and a-modally completes the occluded one, taking both at the same time as perceived units. In the numerical experiment first the image is lifted in the roto-translation space (top right) and the missing information is completed by the algorithm (bottom). The result shows that the partially occluded object has been completed and the occluding one has been segmented. Both the objects are present at the same time in the roto-translation space.

Finally a classical cognitive image, like the Kanizsa fishes, has been considered. This image has been deeply studied in the past because it induces several perception 

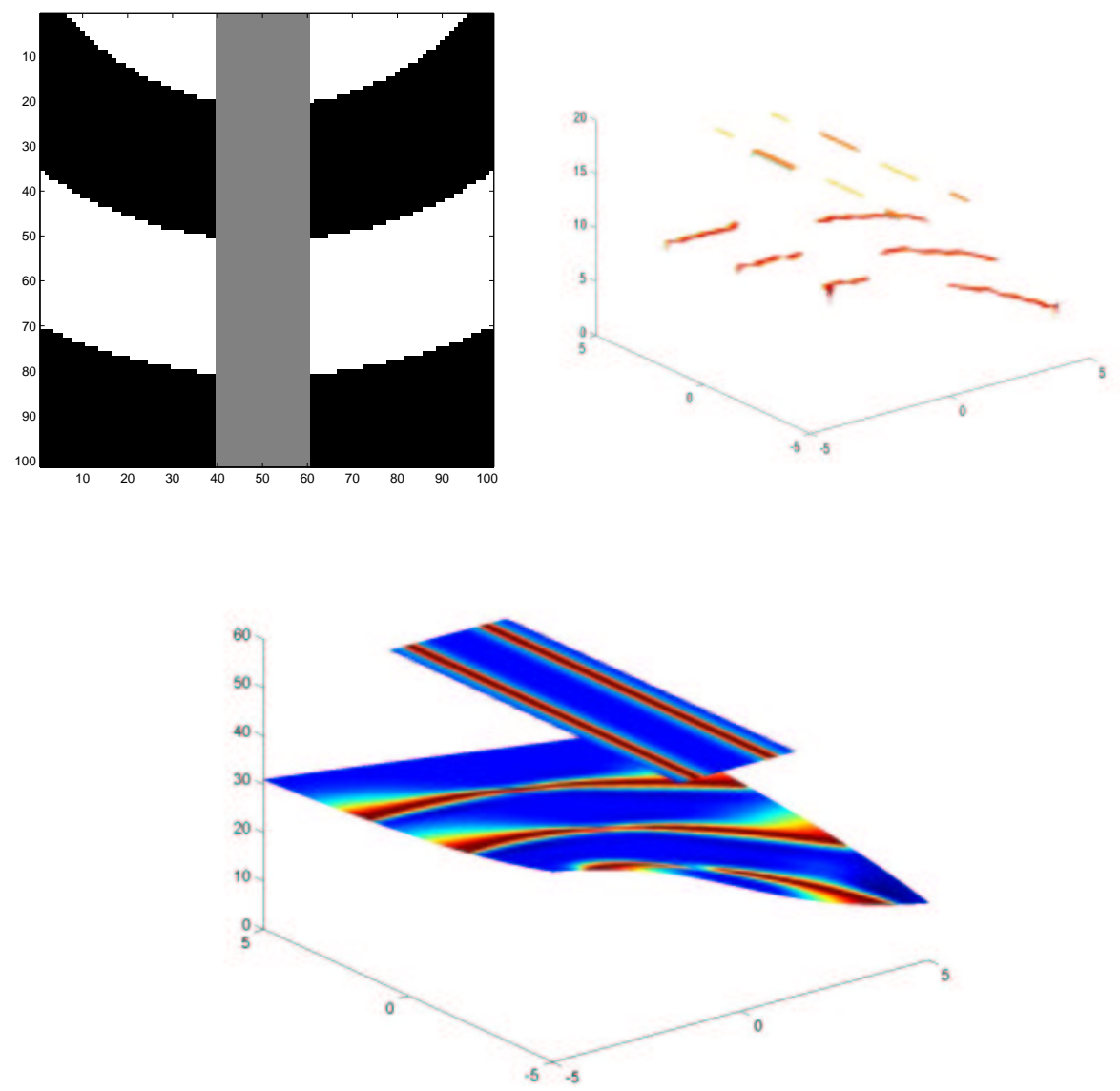

Figure 13. The original image (top left) is lifted in the rototranslation space (top right). Modal and amodal completion are performed at the same time by the algorithm (bottom).

phenomena. First the fishes heads are modally completed and they appear to occlude the tails that are in turn a-modally completed. This double phenomenon does not allow to introduce a depth ordering between the two objects, but it does not prevent a clear perception of the two fishes. The original image (Figure 14 top) and is lifted in the rototranslation space. Modal and amodal completion are performed at the same time by the algorithm. Two different view of the completed image are shown in Figure 14: in the view from the top the modally completed parts are visible (middle). In the view from the bottom the amodally completed parts are shown (bottom). The two fishes are contemporary present in the 3D RT space.

\section{Proof of Convergence}

In this section we give a sketch of the proof of the convergence of our diffusion concentration algorithm. A first relation between diffusion and curvature equation 

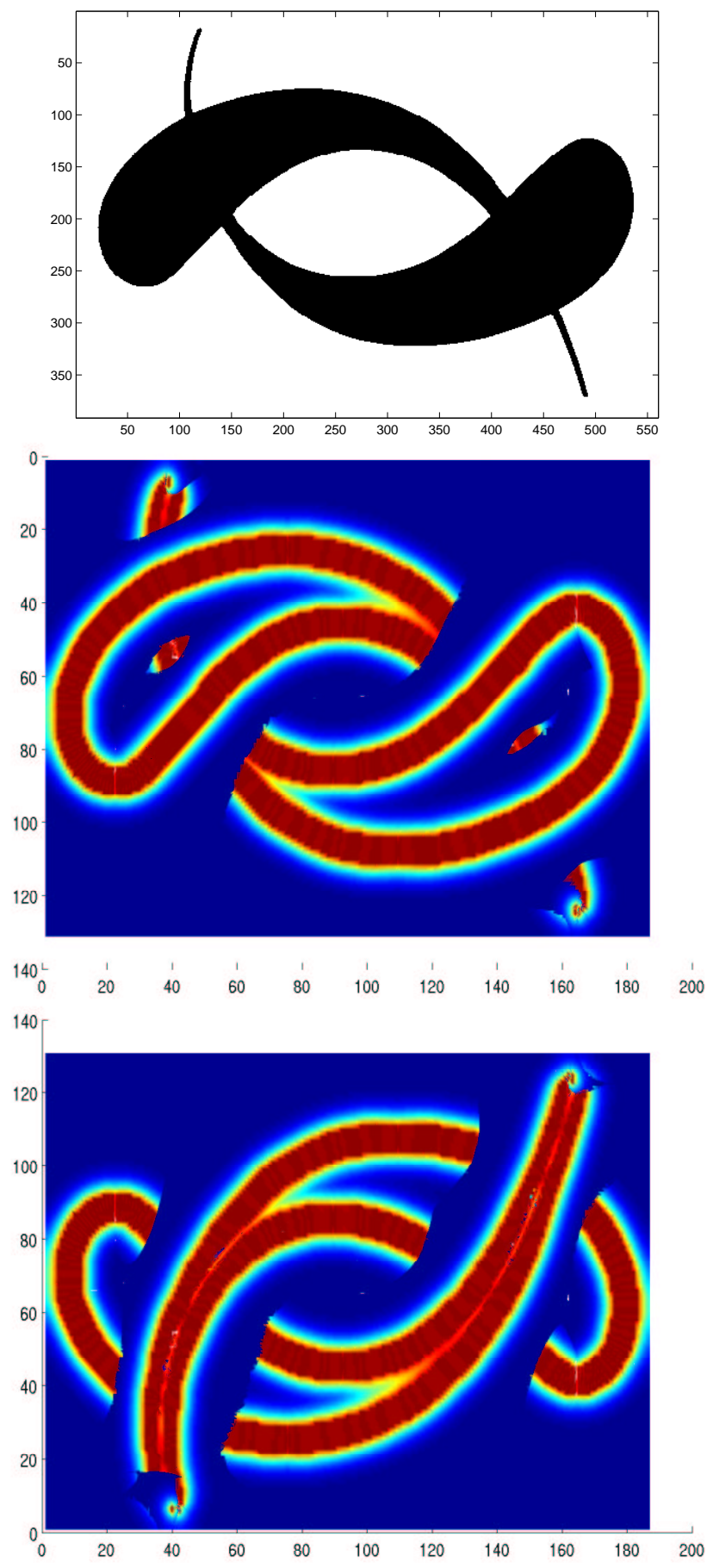

Figure 14. The original image (top) is lifted in the rototranslation space. Modal and amodal completion are performed at the same time by the algorithm. Two different view of the completed image are shown: in the view from the top the modally completed parts are visible (middle). In the view from the bottom the amodally completed parts are shown (bottom). 
goes back to paper of Bence, Merrimann and Osher [7] who describes the evolution of surface by mean curvature in terms of heat diffusion. Formal proof of the convergence of the motion of $\Sigma_{n}$ to the motion by curvature has been provided independently by Evans, [17] and Barles and Georgelin [2]. Here we further develop these ideas, relating the diffusion, with motion by curvature and Laplace beltrami operator on a surface, in the subriemannian setting.

Using the fundamental solution we can represent the solution of the sublaplacian heat equation, with initial datum $u_{0} \delta_{0}$ as an integral on the surface $\Sigma_{0}$ :

$$
\begin{gathered}
u((x, y, \theta), t)=\int_{\Sigma_{0}} \Gamma(\zeta) u_{0}\left(\zeta_{-}(x, y, \theta)\right) d \sigma(\zeta)= \\
=\int_{\Sigma_{0}} \Gamma\left({ }_{R}^{-} \zeta_{R}^{+}(x, y, \theta), t\right) u_{0}(\zeta) d \sigma(\zeta),
\end{gathered}
$$

where $\sigma$ denotes the element of area on the surface $\Sigma_{0}$, and $\zeta$ is a $3 \mathrm{D}$ point of the space. The expression of the fundamental solution is not known explicitly, but it can be approximated locally in time, with the parametrix method of Miranda (see [57], for the application of the method to general subelliptic operators - see also $[8]$ ). Indeed

Theorem 5.1. In a neighborhood of each point $\xi_{0}=\left(x_{0}, y_{0}, \theta_{0}\right)$, there exists a regular change of variables

$$
\left\{\begin{array}{cccc}
x_{1} & = & \left(x-x_{0}\right) \cos \left(\theta_{0}\right)+\left(y-y_{0}\right) \sin \left(\theta_{0}\right), \\
\theta_{1}= & \theta-\theta_{0}, \\
y_{1}= & -\left(x-x_{0}\right) \sin \left(\theta_{0}\right)+\left(y-y_{0}\right) \cos \left(\theta_{0}\right)-\frac{1}{2} x_{1} \theta_{1},
\end{array}\right.
$$

and regular function

$$
\Gamma_{H}((x, y, \theta), t)=\frac{1}{(2 \pi t)^{4}} \int_{R} \cos \left(\frac{y \tau}{t}\right) \exp \left(\left(\frac{1}{2} \tau \operatorname{coth}(2 \tau)\left(x^{2}+\theta^{2}\right)\right) / t\right) \frac{(2 \tau)^{4}}{\sinh (2 \tau)^{4}} d \tau
$$

such that the function

$$
\left.\Gamma_{\xi_{0}}((x, y, \theta), t)=\Gamma_{H}\left(\left(x_{1}, y_{1}, \theta_{1}\right), t\right)\right),
$$

is a parametrix of the fundamental solution. Precisely

$$
\left|X_{2} \Gamma-X_{2} \Gamma_{\xi_{0}}\right|((x, y, \theta), t) \leq(t-\tau)^{-1 / 2} \Gamma_{\xi_{0}}((x, y, \theta), t)
$$

in a neighborhood of the point $\xi$ (a similar assertion also holds for $X_{1}$ ). Besides

$$
\left|\nabla_{X} \Gamma((x, y, \theta), t)\right| \leq \frac{C}{t^{Q / 2}} \exp \left(-\frac{d^{2}(x, y, \theta)}{t}\right)
$$

where $Q=4$. (see [CMS[]] for the proof).

We explicitly remark that the function $\Gamma_{H}$ has the usual homogeneity property:

$$
\Gamma_{H}\left(\delta_{\sqrt{t}}(x, y, \theta), t\right)=\Gamma_{H}((x, y, \theta), 1)
$$

with respect to the non homogeneous dilations defined in (13):

$$
\delta_{\sqrt{t}}(x, y, \theta)=(\sqrt{t} x, t y, \sqrt{t} \theta) .
$$


In order to show that the surface is moving by curvature, we take $\left(x_{0}, y_{0}, \theta_{0}\right) \in \Sigma_{0}$ and denote $\nu=\nu_{0}$ the normal to $\Sigma$ at $\left(x_{0}, y_{0}, \theta_{0}\right)$. Then we select $v \in \mathbb{R}$ so that $\left(x_{0}, y_{0}, \theta_{0}\right)_{R}+t v \nu \in \Sigma_{1}$, in other words:

$$
\partial_{\nu} u\left(\left(x_{0}, y_{0}, \theta_{0}\right)+\underset{R}{+} t v \nu, t\right)=0
$$

Then $v t$ is the normal increment from $\left(x_{0}, y_{0}, \theta_{0}\right)$ to the new surface $\Sigma_{1}$. We have to prove the following theorem

\section{Theorem 5.2.}

$$
v=H\left(x_{0}, y_{0}, \theta_{0}\right)+o(1)
$$

as $t \rightarrow 0$, where $H$ is the curvature of the initial surface at the point $\left(x_{0}, y_{0}, \theta_{0}\right)$.

Proof Up to a change of coordinates, we may assume that $\left(x_{0}, y_{0}, \theta_{0}\right)=0$, $\nu=(0,1,0)$, and $\partial_{\nu}=X_{2}$, and $\Sigma$ is a graph:

$$
\left\{\theta=h(x, y):(x, y) \in Q^{\prime}\right\}
$$

for some function $h$, and a suitable cube $Q^{\prime}$. We then have

$$
h(0)=0, X_{1} h(0)=0, X_{1}^{2} h(0)=\operatorname{curv}(0) .
$$

Calling $s=\left(x_{0}, y_{0}, \theta_{0}\right)+{ }_{R} t v \nu=(0,0, t v)$ and differentiating the expression of $u$ we get

$$
0=X_{2} u(s)=\int_{\Sigma} X_{2} \Gamma\left(\underset{R}{-} \zeta_{R} s, t\right) d \sigma(\zeta)=
$$

where we have denoted $\zeta=(x, y, \theta)$

$$
=\int_{\Sigma \cup C} X_{2} \Gamma\left({ }_{R} \zeta_{+} s, t\right) d \sigma(\zeta)+O\left(e^{-t}\right)=
$$

using the approximation in Theorem, and denoting $\zeta_{1}$ the image of $\zeta$ in the change of variable,

$$
=\int_{\Sigma \cup C} X_{2} \Gamma_{H}\left({ }_{R}^{-} \zeta_{1}+s, t\right) u(\zeta) d \sigma(\zeta)+O\left(e^{-t}\right)=
$$

(the derivative of $\Gamma_{H}$ is of the form $\theta K$, for a suitable kernel never vanishing $K$, exponentially decaying, and satisfying 20 ).

$$
=\int_{\Sigma \cup C} \frac{1}{(2 \pi t)^{4}} \frac{(\theta-v t)}{t} K\left({ }_{R} \zeta_{1}+s, t\right) d \sigma(\zeta)+O\left(e^{-t}\right)
$$

Since the integral is performed on the surface $\Sigma$, graph of $h$

$$
=\int_{C} \frac{1}{(2 \pi t)^{4}} \frac{(h(x, y)-v t)}{t} K\left({ }_{R} \zeta_{1}{ }_{R} s, t\right) \sqrt{1+|\nabla h(x, y)|^{2}} d x d y+O\left(e^{-t}\right)
$$

With the change of variable $x=\sqrt{t} p y=t q$,

$$
=\int_{C} \frac{1}{(2 \pi t)^{4}} \frac{(h(\sqrt{t} p, t q)-v t)}{t} K\left({ }_{R}^{-} \zeta_{1}{ }_{R}^{+} s, t\right) \sqrt{1+|\nabla h(\sqrt{p}, t q)|^{2}} d x d y+O\left(e^{-t}\right)
$$

Since this expression is 0 , the first non zero term of the Taylor development of

$$
v t-h(\sqrt{t} p, t q)
$$

must vanishes. Since the first derivative of $h$ is 0 , this implies that

$$
v=H\left(x_{0}, y_{0}, \theta_{0}\right)+O(\sqrt{t}) .
$$


We explicitly note that this is only a local approximation result. However the convergence proof performed by [2], [17], are based on the semigroup theory, and can be repeated in the rototraslation one. We refer to [11] for details.

\section{REFERENCES}

[1] L. Ambrosio, S. Masnou, A direct variational approach to a problem arising in image recostruction, preprint

[2] G.Barles, C. Georgelin, A simple proof for the convergence for an approximation wcheme for computing motions by mean curvature, SIAM J. Numerical Analysis, 32, (1995), 484-500.

[3] O. Bar and H.Sompolinsky, R. Ben-Yishai. Theory of orientation tuning in visual cortex, Proc. Natl. Acad. Sci. U.S.A., 92: 3844-3848, 1995

[4] A. Bellaiche, The tangent space in sub-Riemannian geometry. Proceedings of the satellite meeting of the 1st European congress of mathematics 'Journes nonholonomes: gomtrie sousriemannienne, thorie du contrle, robotique', Paris, France, June 30-July 1, 1992. Basel: Birkhuser. Prog. Math. 144, 1-78 (1996).

[5] G. Bellettini, R. March, An image segmentation variational model with free discontinuities and contour curvature, preprint.

[6] C. Ballester, M. Bertalmio, V. Caselles, G. Sapiro, J. Verdera, Filling-in by interpolation of vector fields and gray levels, preprint.

[7] J. Bence, B. Merriman, S. Osher, Diffusion generated motion by mena curvature, in Computational Crystal Growers Workshop, J. Taylor Sel. Taylor Ed.,

[8] A.Bonfiglioli, E.Lanconelli, F. Uguzzoni, Fundamental solutions for non-divergence form operators on stratified groups to appear in Trans. Amer. Math. Soc.

[9] L.Capogna, D.Danielli, N.Garofalo, The geometric Sobolev embedding for vector fields and the isoperimetric inequality, Comm Anal. Geo. 12, (1994), 203-215.

[10] M. Carandini and D.L. Ringach. Predictions of a recurrent model of orientation selectivity. Vision Res., 37: 3061-3071, 1997

[11] G. Citti, M. Manfredini, A. Sarti, Neuronal Oscillations in the Visual Cortex: $\Gamma$-convergence to the Riemannian Mumford-Shah Functional, preprint.

[12] J.G.Daugman Uncertainty - relation for resolution in sapce, spatial frequency and orientation optimized by two dimensional visual cortical filters, J. Opt. Soc. Amer,. 2(7), 1160-1169, 1985

[13] E. De Giorgi, Some remarks on $\Gamma$ convergence and least square methods in "Composite media and homogeniziation Theory, G. Dal Masoand G. F. Dell'Antonio (Eds.), Birkhauser Boston, 1991, 153-142.

[14] S. Esedoglu, R. March, Segmentation with Deph but without detecting junctions, Journal of Mathematical Imaging and Vision, 18, 7-15, 2003.

[15] A.K.Engel, P.Konig, C.M.Gray and W.Singer, Stimulus dependent neuronal oscillations, in cat visual cortex: intercolumnar interaction as determined by cross-correlation analysis. European Journal of Neuroscience, 2, pag 558-606, 1990

[16] A.K.Engel, A.K. Kreiter, P.Konig and W.Singer, Syncronization of oscillatory neuronal responses between striate and extrastriate visual cortical areas of the cat, PNAS, 88, 6048-6052. D.J.

[17] L. Evans, Convergence of an Algorithm for mean curvature motion, Indiana Univ. Math J., vol 42, n. 2, 553-557, 1993.

[18] B. Franchi, R. Serapioni, F. Serra Cassano, On the structure of finite perimeter sets in step 2 Carnot groups, preprint.

[19] G.B. Folland, Subelliptic estimates and function spaces on nilpotent Lie groups, Ark. Mat. 13, (1975), 161-207.

[20] G.B. Folland, On the Rothschild-Stein lifting theorem, Commun. Partial Differ. Equations 2, (1977), 165-191.

[21] G.B. Folland, E.M. Stein, Estimates for the $\bar{\partial}_{b}$ Complex and Analysis on the Heisenberg Group, Comm. Pure Appl. Math. 20, (1974), 429-522.

[22] R. Goodman, Lifting vector fields to nilpotent Lie groups J. Math. Pures Appl., IX. Sr. 57, (1978), 77-85.

[23] C.D. Gilbert, A. Das, M. Ito, M.Kapadia, G. Westheimer, Spatial integration and cortical dynamics. Proceedings of the National Academy of Sciences USA, 93, pag 615-622 
[24] C.M. Gray, P.Konig, A.K.Engel, W. Singer, Oscillatory responses in cat visual cortex exhibit inter-columnar syncronization which reflects global stimulus properties, Nature, 338, pag. 334-337, 1989

[25] S.Grossberg and E.Mingolla Neural dynamics of perceptual grouping: textures, boundaries and emergent segmentations, in "Perception and Psychophysics", 1985

[26] Field, A.Heyes, R.F. Hess, Contour integration by the human visual system: evidence for a local Association Field, Vision Research, 33, pag 173-193, 1993

[27] W.C. Hoffman, The visual cortex is a contact bundle, Applied Mathematics and Computation, vol 32, (89), 137-167.

[28] W.C. Hoffman, M. Ferraro. Lie transformation groups, integral transforms, and invariant pattern recognition. Spatial Vision 8 (1994), 33-44.

[29] H. Hörmander, Hypoelliptic second-order differential equations, Acta Math., 119, (1967), $147-171$.

[30] H. Hörmander, A. Melin, Free systems of vector fields, Ark. Mat, 16 (1978), 83-88.

[31] Hubel, D. Wiesel, T. (1962). Receptive fields, binocular interaction and functional architecture in the cat's visual cortex. Journal of Physiology, 160, 106154.

[32] J.P Jones and L.A.Palmer An evaluation of the two-dimensional gabor filter model of simple receptive fields in cat striate cortex, J. Neurophysiology, 58:1233-1258, 1987

[33] Kanizsa G., Grammatica del vedere, Il Mulino, Bologna, 1980.

[34] Kanizsa G., Organization in Vision, Hardcover, 1979.

[35] M.K.Kapadia, M.Ito, C.D.Gilbert, G.Westheimer, Improvement in visual sensitivity by changes in local context: parallel studies in human observers and in V1 of alert monkeys, Neuron, 15, pag 843-856,1995

[36] S. Kusuoka and D. Stroock, Applications of the Malliavin calculus III, J. Fac. Sci. Univ. Tokio, Sect. IA, Math, 34, 391-442, 1987.

[37] S. Kusuoka and D. Stroock, Long time estimates for the heat kernel associated with a uniformly subelliptic symmetric second order operator, Ann. of Math. 127, 165-189, 1988.

[38] I.Kovacs, B. Julesz, A closed curve is much more than an incomplete one: effect of closure in figure-ground segmentation, PNAS, 90, pag 7495-7497,1993

[39] I.Kovacs, B.Julesz, Perceptual sensitivity maps within globally defined visual shapes, Nature, 370, pag. 644-646,1994

[40] LeVeque, Randall, J. Nonlinear conservation laws and finite volume methods. (English) Steiner, Oskar et al., Computational methods for astrophysical fluid flow. Saas-Fee advanced course 27. Lecture notes 1997. Swiss Society for Astrophysics and Astronomy. Berlin: Springer. 1-159 (1998)

[41] S.Marcelja, Mathematical description of the response of simple cortical cells, J. Opt. Soc. Amer.70, pag. 1297-1300, 1980

[42] V. Magnani, Differentiability and area formula on stratified Lie groups, Houston J. Math. 27, No.2, (2001), 297-323.

[43] J. Manfredi, Fully non linear subelliptic equations, preprint.

[44] Mumford, Nitzberg Shiota, Filtering, Segmentation and Deph, Springer-Verlag, Berlin, 1993.

[45] S. Masnou, J.M. Norel, "Level lines based disocclusion", Proc. 5th. IEEE International Conference on Image Processing, Chicago, Illinois, October 4-7, 1998.

[46] K.D. Miller, A. Kayser, N. J. Priebe, "Contrast-dependent nonlinearities arise locally in a model of contrast-invariant orientation tuning", J. Neurophysiol., 85: pag.2130-2149, 2001

[47] E. Mingolla, Le unità della visione, IX Kanitza lecture, Trieste symposium on perception and cognition, 26 october 2001.

[48] A. Nagel, E.M. Stein, S. Wainger, Balls and metrics defined by vector fields I: Basic properties, Acta Math. 155, (1985), 103-147.

[49] S.B. Nelson and M. Sur D.C. Somers. An emergent model of orientation selectivity in cat visual cortical simples cells, J. Neurosci., 15: 5448-5465, 1995

[50] S.D. Pauls, A notion of rectifiability modeled on Carnot groups, preprint.

[51] S.D. Pauls, minimal surfaces in the Heisenberg group, preprint.

[52] P. Perona, Deformable kernels for early vision. IEEE-PAMI, 17(5):488-499, 1995.

[53] Petitot J., Phenomenology of Perception, Qualitative Physics and Sheaf Mereology, Proceedings of the 16th International Wittgenstein Symposium, Vienna, Verlag Hlder-PichlerTempsky (1994) 387-408. 
[54] Petitot J., Tondut Y., Vers une Neuro-geometrie. Fibrations corticales, structures de contact et contours subjectifs modaux, Mathmatiques, Informatique et Sciences Humaines, EHESS, Paris, 145 (1998) 5-101.

[55] Petitot J., Morphological Eidetics for Phenomenology of Perception, in Naturalizing Phenomenology: Issues in Contemporary Phenomenology and Cognitive Science, ( Petitot J., Varela F.J., Roy J.-M., Pachoud B. , eds.), Stanford, Stanford University Press, (1998), 330-371.

[56] N.J. Priebe and K.D. Miller T.W. Troyer, A.E. Krukowsky. Contrast-invariant orientation tuning in cat visual cortex: thalamocortical input tuning and correlation-based intracortical connectivity. J. Neurosci., 18: 5908-5927, 1998

[57] L. Rothschild, E.M. Stein, Hypoelliptic differential operators and nihilpotent Lie groups, Acta Math. 137, (1977), 247-320.

[58] A. Sarti, R. Malladi, J.A. Sethian, Subjective surfaces: A Method for Completion of Missing Boundaries, Proceedings of the National Academy of Sciences of the United States of America, Vol 12, N.97, pag. 6258-6263, 2000.

Published online before print May 23, 2000: http://www.pnas.org/cgi/content/full/110135797

[59] A. Sarti, G. Citti, M. Manfredini, From neural oscillations to variational problems in the visual cortex, invited paper.

[60] M. Shelley and D. J. Wielaard D. McLaughlin, R. Shapley. A neuronal network model of macaque primary visual cortex (v1): orientation selectivity and dynamics in the input layer 4calpha. Proc. Natl. Acad. Sci. U.S.A., 97: 8087-8092, 2000

[61] Shih-Cheng Yen, Leif H. Finkel, Extraction of Perceptually Salient Contours by Striate Cortical Networks, Vision Res., Vol. 38, N. 5, pag 719-741, 1998

[62] Y.-Q. Song, X.-P. Yang, BV function in the Heisenberg group, preprint.

[63] E.M. Stein, Harmonic Analysis, Princeton University Press, (1993).

[64] Vodop'yanov, S.K.; Ukhlov, A.D. Approximately differentiable transformations and change of variables on nilpotent groups. Sib. Math. J. 37, No.1, 62-78 (1996); translation from Sib. Mat. Zh. 37, No.1, (1996), 70-89.

[65] V.S. Varadarajan, Lie groups, Lie algebras, and their representations, Graduate Texts in Mathematics. 102, New York, Springer (1984).

[66] N.T. Varopoulos, L. Saloff-Coste, and T. Coulhon, Analysis and geometry on groups Cambridge texts in Mathematics 100, cambridge University Press, Cambredge, 1992.

[67] Frank W. Warner, Foundations of differentiable manifolds and Lie groups. (English) Glenview, Illinois-London: Scott, Foresman \& Comp. 270 p. 4.24 (1971).

[68] F. Worgotter, C. Koch, "A detailed model of the primary visual pathway in the cat: comparison of afferent excitatory and intracortical inhibitory connection schemes for orientation selectivity. J. Neurosci., 11: 1959-1979, 1991 